\title{
Two-Dimensional Nanoscale Self-Assembly on a Gold Surface by Spinodal Decomposition
}

\author{
Rolf Schuster, ${ }^{*}$ Dominik Thron, Marcello Binetti, Xinghua Xia, ${ }^{\dagger}$ and Gerhard Ertl \\ Fritz-Haber-Institut der Max-Planck-Gesellschaft, Faradayweg 4-6, D-14195 Berlin, Germany
}

(Received 28 February 2003; published 5 August 2003)

\begin{abstract}
The structure formation upon spinodal decomposition of a two-dimensional model system, a Au adatom gas on a $\mathrm{Au}(111)$ surface, was observed in situ by scanning tunneling microscopy (STM). A thermodynamically unstable state was prepared by applying microsecond voltage pulses to the STM tip in an electrochemical system, causing the random dissolution of $\mathrm{Au}$ atoms from the uppermost monolayer. Interconnected, labyrinthine island patterns were formed at Au coverages between 0.4 and 0.9 monolayer with dominating length scales $\lambda_{\mathrm{m}}$ of the order of a few nanometers.
\end{abstract}

DOI: 10.1103/PhysRevLett.91.066101

The evolution of structure out of a homogeneous system undergoing phase separation is a common phenomenon, encountered upon the condensation of water droplets in clouds [1] as well as upon metal deposition on surfaces [2]. From experience such first order phase transitions are mostly governed by the formation of compact shapes with minimized phase boundary energy, as a consequence of the formation of stable nuclei and their subsequent growth. However, as already pointed out by Gibbs, upon penetrating deeply enough into the coexistence region, the activation barrier against the formation of stable nuclei vanishes and the system becomes unstable. Because of the absence of any activation barrier the instantaneous, i.e., spinodal, decomposition of the system proceeds very fast, with density fluctuations of a certain wavelength growing exponentially with time [3]. This wavelength is determined solely by the thermodynamic properties of the system [4]. When the volume fractions of the phases of the heterogeneous system are close to $50 \%$, labyrinthine interconnected patterns evolve, distinctively different from the morphologies expected upon nucleation and growth.

In this Letter we demonstrate how the thermodynamic stability of a surface system can be employed to steer the morphology of resulting patterns from growth of compact islands to labyrinthine interconnected structures on the nanoscale. Phase transitions in a two-dimensional (2D) model system, a gas of $\mathrm{Au}$ adatoms on a $\mathrm{Au}(111)$ surface, are induced by electrochemical, randomly distributed dissolution of $\mathrm{Au}$ atoms out of the topmost layer of a single crystal surface. This process occurs during a voltage pulse of only microsecond duration applied to the tip of an electrochemical scanning tunneling microscope (STM) facing the sample surface. This period is short enough to avoid significant diffusion and ordering already during the change of the state of the system. The resulting atomic-scale morphology is then imaged in situ by STM. The simplicity of the system justifies its description by a 2D lattice gas model with pairwise nearest neighbor interactions. Therefore, the system comes close to the models considered in the very first theoretical treatments
PACS numbers: 64.75.+g, 05.70.-a, 68.37.Ef, 82.45.Qr

of spinodal decomposition by Cahn and Hilliard $[4,5]$ and the evolving structures can be directly compared to the theoretical predictions, derived explicitly for lattice model systems.

To induce the phase separation in the Au surface system, we chose electrochemical dissolution of $\mathrm{Au}$ atoms in a $2 \mathrm{M}$ aqueous $\mathrm{KCl}$ electrolyte (Merck, suprapure), since electrochemical reactions can be driven extremely fast at sufficiently high overpotentials and the Au dissolution is not hampered by diffusion limitations in the bulk of the electrolyte. Complex formation due to the presence of chloride ions allows for the direct dissolution of Au atoms out of the Au topmost layer. To avoid the finite charging time constant of conventional electrochemical cells (typically milliseconds) [6], the STM tip itself was employed as local counter electrode. After gating the STM feedback loop, the STM tip was retracted by a few $\mathrm{nm}$, before positive rectangular voltage pulses of $\mu$ s duration were applied between the Au sample and the tip. This leads to the dissolution of randomly distributed $\mathrm{Au}$ atoms from the topmost surface layer. The remaining $\mathrm{Au}$ atoms constitute a 2D lattice gas on a $\mathrm{Au}(111)$ surface, whose ordering into stable phases was observed in situ by moving the STM tip back in tunneling position immediately after the pulse. The interruption of the imaging mode took only about $3 \mathrm{~ms}$ and did not interfere with the scanning of the tip, since in the fast scan direction the recording of a single line of the STM image took $180 \mathrm{~ms}$. The stationary potentials of the Au sample and the STM tip were controlled by a bipotentiostat versus a $\mathrm{Ag} / \mathrm{AgCl}$ reference electrode (for further details, see [7]). Evaporated $\mathrm{Au}$ films on glass were used as samples, which were cyclically rinsed with distilled water (Millipore Q) and flame annealed several times to obtain single crystalline $\mathrm{Au}(111)$ terraces extending up to several $100 \mathrm{~nm}$.

Figure 1(a) shows the result of a single $0.7 \mu \mathrm{s}, 5.2 \mathrm{~V}$ pulse applied to the scanning STM tip at the position marked by the arrow, the slow scan direction pointing downwards. Immediately after the pulse a labyrinthine pattern of monoatomically high $\mathrm{Au}$ islands appeared on the surface, which coarsened slightly while image 

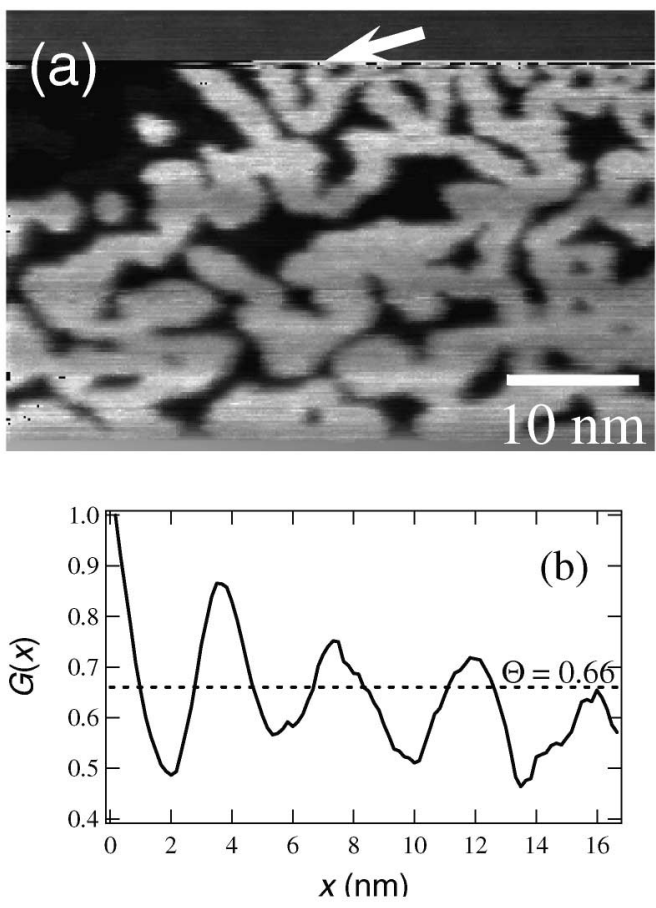

FIG. 1. (a) In situ STM image of the evolution of a labyrinthine, interconnected island pattern upon the application of a $0.7 \mu \mathrm{s}, 5.2 \mathrm{~V}$ pulse between the STM tip and the $\mathrm{Au}(111)$ surface. The position of the STM tip, where the pulse was applied, is indicated by an arrow. The slow scan direction was directed downwards. The island pattern significantly coarsened during the imaging at a potential of $\phi_{\mathrm{Ag} / \mathrm{AgCl}}=0.4 \mathrm{~V}$. (b) Linewise density autocorrelation function of the STM image in (a), $1 \mathrm{~s}$ after the pulse.

scanning proceeded. Diffusion of Au atoms and consequent coarsening of the structures can be suppressed by lowering the potential of the $\mathrm{Au}$ sample, as discussed later (see Fig. 2). Because of current density limitations, the electrochemical reactions were confined to an area of about $100 \mathrm{~nm} \times 100 \mathrm{~nm}$ close to the tip.

To quantify typical length scales of the surface morphology, the linewise density autocorrelation function $G(x)$ of Fig. 1(a) was calculated $1 \mathrm{~s}$ after the pulse according to $G(x)=\langle\rho(s) \rho(s+x)\rangle$, where $\rho=1$ on the $\mathrm{Au}$ islands and $\rho=0$ in between [Fig. 1(b)]. $G(x)$ clearly exhibits oscillations, signaling a dominating wavelength of the pattern of about $4 \mathrm{~nm}$. The half width of $G(x)$ ), which provides the average island width along the fast scan direction, amounts to about $2 \mathrm{~nm}$. The average $\mathrm{Au}$ atom density, i.e., coverage was evaluated to $\theta=0.66$ $(\theta=1$ corresponds to a complete $1 \times 1$ layer of $\mathrm{Au})$. Because of the rather sharp island edges, both the half width of the correlation function and its periodicity were rather insensitive with respect to the threshold for $\mathrm{Au}$ island identification.

Morphologies similar to Fig. 1(a) are usually not observed for surface systems prepared, for example, by metal on metal deposition or adsorption from the gas

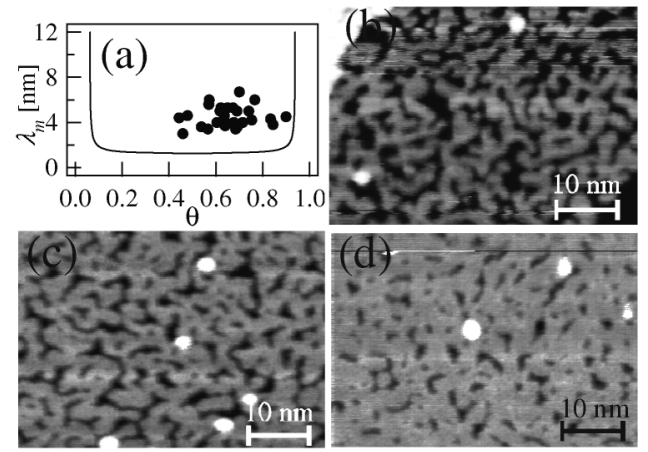

FIG. 2. (a) Solid line: dominant wavelength $\lambda_{\mathrm{m}}$, expected from Cahn and Hilliard theory for the spinodal decomposition of a $2 \mathrm{D}$ Au adatom gas. The circles summarize experimental results for the dominating wavelengths, following pulses with different duration and hence differing Au coverage. (b)(d) Surface morphology following $5 \mathrm{~V}$ pulses of $2.8 \mu \mathrm{s}$ (b), $2.1 \mu \mathrm{s}(\mathrm{c})$, and $0.7 \mu \mathrm{s}(\mathrm{d})$ duration. Au densities are $\theta=0.5$, $\theta=0.7$, and $\theta=0.8$. Irrespective of the surface coverage, the dominating wavelength of the structure amounts to about $4 \mathrm{~nm}$.

phase. In those cases the growth of the new phase starts already during the deposition process, due to the high mobility of the adatoms compared with the time necessary for an appreciable variation of the coverage (typically minutes). The observed morphologies are hence governed by nucleation and growth, and rather compact island structures are usually found already at the very beginning of the deposition process [2]. To suppress diffusion of adatoms and subsequent structure formation during the deposition process, Ernst et al. performed deposition of $\mathrm{Cu}$ on $\mathrm{Cu}(100)$ at low temperature [8]. Following an upward temperature quench, the formation of interconnected structures from a quasirandom initial adlayer state was inferred from He atom beam scattering data reflecting the progress of ordering. Although the initial length scale of the observed patterns was on the same order of magnitude as in the present experiments, the real space morphology of the structures could not be resolved. Interconnected domain patterns were also observed upon homoepitaxy on $\mathrm{Ag}(100)$. In those cases they were explained by a nucleation and growth mechanism, which was followed by diffusion-mediated coalescence of $\mathrm{Ag}$ clusters [9]. Because of the dominant nucleation and growth mechanism, these structures are much less ramified than those of Fig. 1(a) and interconnected domain patterns formed only at fairly high coverages. Furthermore, typical length scales exceeded $30 \mathrm{~nm}$.

Different from conventional deposition of adatoms, the electrochemical removal of atoms, i.e., the formation of $\mathrm{Au}$ vacancies at the surface, allows a change of the thermodynamical state within microseconds. This is fast enough to avoid significant ordering during the dissolution process so that characteristic labyrinthine patterns can evolve. Indeed, the activation energy for the diffusion of $\mathrm{Au}$ vacancies in the topmost terrace was derived to 
$0.46 \mathrm{eV}$ [10], resulting in a hopping rate of one jump per $10 \mu \mathrm{s}$ at room temperature, if a prefactor of $10^{13} \mathrm{l} / \mathrm{s}$ is employed.

Although the evolution of a labyrinthine pattern "with its high degree of connectivity is also a good indication that a spinodal mechanism is involved" [4], this has to be further substantiated. For a theoretical analysis of the present system, we closely followed the procedure given by Cahn and Hilliard in their seminal papers [4,5]. According to this theory, the decomposition process is dominated by density fluctuations with a wave vector $\beta_{\mathrm{m}}=\frac{1}{2}\left[-\left(\partial^{2} f / \partial c^{2}\right) / \kappa\right]^{1 / 2}$, where $f$ is the Free energy of the homogeneous system as a function of the concentration $c$ of one component, i.e., the Au atom coverage. The factor $\kappa$ represents the range and magnitude of the interaction energies of the mixture's components. For simplicity we assumed that the $\mathrm{Au}$ interactions are dominated by nearest neighbor two-body interactions. This allows the straightforward translation of the onecomponent lattice gas system into the notation for a twocomponent mixture, which is used by Cahn and Hilliard. Slightly modifying the calculation of $\kappa$ in Ref. [5] for a 2D adatom gas on a hexagonal lattice with six nearest neighbors and treating the free energy in the BraggWilliams approximation of a lattice gas [11] leads to a dominating wave vector $\beta_{\mathrm{m}}=1 / r\left[2+k_{\mathrm{B}} T / 3 \omega \theta(1-\right.$ $\theta)]^{1 / 2}$, with the nearest neighbor distance $r$ and the pair interaction energy $\omega$ between the Au atoms. $\theta$ denotes the average $\mathrm{Au}$ adatom density, $k_{\mathrm{B}}$ the Boltzmann constant, and $T$ the temperature. Au adatoms at room temperature exhibit a strong tendency for the formation of compact $(1 \times 1)$ terraces, indicating a critical temperature far above room temperature and a pair interaction energy exceeding several $k_{\mathrm{B}} T$. A rough estimate on the pair interaction energy in an electrochemical surrounding similar to our conditions can be inferred from step fluctuation measurements on $\mathrm{Au}(111)$ surfaces immersed in chloride containing electrolytes [12]. There the kink formation energy corresponding to breaking a single bond at the edge of a monoatomic, close packed step was estimated to $74 \mathrm{meV}$, leading to a wavelength $\lambda_{\mathrm{m}}=$ $2 \pi / \beta_{\mathrm{m}}=1.5 \mathrm{~nm}$. Theoretical calculations within the effective medium theory yielded a formation energy for an adatom-vacancy pair at a $\mathrm{Au}(111)$ terrace of $1 \mathrm{eV}$ [10], resulting in an energy per bond of $0.17 \mathrm{eV}$ and a corresponding wavelength of $1.1 \mathrm{~nm}$.

The linearized theory of Cahn and Hilliard describes properly the early stages of spinodal decomposition. At later stages, i.e., upon the formation of a pronounced island pattern, the inclusion of nonlinear terms in the derivative of the free energy becomes necessary. Bortz et al. pointed out that particularly in systems with short range interactions coarsening sets in immediately at the beginning of the phase separation [13]. This leads to a continuous increase of the wavelength with progressing decomposition already during the early stages of the decomposition process. This finding is in agreement with calculations for critical quenches by Langer and coworkers [14], where the dominating wavelength quickly adjusted to about double of what is predicted by the theory above. If this effect is taken into consideration, the experimental finding of a typical wavelength of $4 \mathrm{~nm}$ shortly after the pulse can be nicely correlated with the above theoretical expectations. The continuum theory of Cahn and Hilliard reasonably well describes the evolution of the surface morphology, although the island width is only a few atom diameters wide. It should be noted that in a study of $\mathrm{Ge}$ on $\mathrm{Au}(111)$ in molten salt electrolytes labyrinthine structures with typical island width of $2 \mathrm{~nm}$ were observed by STM in real space [15]. Although the detailed structure and ordering mechanism remained unclear in this study, the island morphology might well result from a spinodal decomposition mechanism. Additionally, the typical wavelength found for the (threedimensional) decomposition of alloys is also in the range of about $5 \mathrm{~nm}$ [16]. This is conceivable since both the lattice parameters and the approximate magnitude of the particle interaction energies in alloy systems are of the same order of magnitude as in our surface system.

According to the linearized theory, at low temperature the prominent wavelength for the spinodal decay should be only weakly dependent on the concentration. The solid line in Fig. 2(a) is calculated by the above formula for a pair interaction energy of $-74 \mathrm{meV}$ at room temperature. To experimentally test this prediction, we varied the average density of the Au adatom gas by changing the duration of the pulse. Figures 2 (b) -2 (d) show the results of $2.8,2.1$, and $0.7 \mu \mathrm{s}, 5 \mathrm{~V}$ voltage pulses, which led to average Au atom densities of $\theta=0.5, \theta=0.7$, and $\theta=$ 0.8 , respectively. (To achieve 2.8 or $2.1 \mu$ s pulse duration, four or three pulses of $0.7 \mu$ s were applied, separated by $3.5 \mu$ s.) In contrast to Fig. 1(a) coarsening of the structures was suppressed by reducing the potential from $\phi_{\mathrm{Ag} / \mathrm{AgCl}}=0.4$ to $0.2 \mathrm{~V}, 100 \mathrm{~ms}$ after the pulses, i.e., within less than one scan line. This significantly reduced the mobility of the Au surface atoms [12]. The time lag of $100 \mathrm{~ms}$ ensured that the dissolved $\mathrm{Au}$ atoms diffused far away into the solution, enough to avoid significant $\mathrm{Au}$ redeposition inside the area of the STM image. The few defects in Figs. 2(b)-2(d) are interpreted as Au islands stemming from such redeposition. At $\theta=0.5$ and $\theta=$ 0.7 labyrinthine island patterns formed similar to Fig. 1(a), whereas at $\theta=0.8$ holes are visible. Already from visual inspection of the STM images typical length scales are very similar to that of the labyrinthine pattern in Fig. 1(a), shortly after the pulse. This is substantiated by the existence of maxima around $4 \mathrm{~nm}$ in the $G(r)$ calculated for Fig. 2. The exact data for the wavelengths together with the results of in total 29 experiments at different coverages are summarized and included in Fig. 2(a). Although the absolute value of the typical length scale is underestimated by Cahn's linearized 


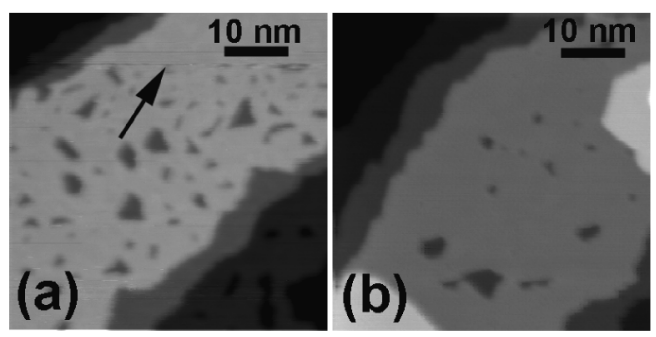

FIG. 3. Slow dissolution of $\mathrm{Au}$ (a) upon application of a $15 \mathrm{~ms}$, $2.4 \mathrm{~V}$ pulse to the STM (tip position during the pulse marked by an arrow) and (b) upon three subsequent $20 \mathrm{~ms}$ potentiostatically controlled potential steps to $\phi_{\mathrm{Ag} / \mathrm{AgCl}}=0.9 \mathrm{~V}$. Well separated holes with diverse diameters, indicative of nucleation and growth processes formed on the surface.

theory, the effective independence of the wavelength on the $\mathrm{Au}$ atom density over a wide coverage range is in agreement with its predictions.

Coverages significantly below $\theta=0.5$ could not be achieved in our experiments even with substantially longer pulses up to several $10 \mu \mathrm{s}$. This can be rationalized considering that prior to the pulse the surface is covered by about half a monolayer of chloride [17], which is consumed by complex formation during the dissolution of $\mathrm{Au}$ and cannot be regenerated during the pulse duration. Furthermore, the terrace step positions remain unchanged upon the pulse application, signaling that $\mathrm{Au}$ is preferentially dissolved by vacancy formation on the terraces and not by dissolution of weakly bound $\mathrm{Au}$ from kink sites at step edges or vacancy clusters.

A high rate of change of the system's state is of crucial importance for the formation of spinodal patterns. With the system investigated here slow dissolution of $\mathrm{Au}$ atoms is expected to lead to nucleation of vacancies and to formation of compact holes already during the dissolution process, before the final $\mathrm{Au}$ atom density is reached. To test this conclusion, we dissolved Au atoms by $15 \mathrm{~ms}$, $2.4 \mathrm{~V}$ voltage pulses to the STM tip and by potentiostatically controlled $60 \mathrm{~ms}$ long potential steps (Fig. 3), i.e., about 4 orders of magnitude more slowly than during the microsecond pulses to the STM tip in the previous experiments. Although in Fig. 3(a) the Au coverage of the topmost terrace amounts to about $\theta=0.75$, in contrast to Fig. 2(c) monoatomically deep holes with widely spread distance and size distributions and mostly close packed boundaries are observed. Between Fig. 3(b) and Fig. 2(d) (with comparable coverage) the difference is even more pronounced. We interpret the morphology seen in Fig. 3 as the consequence of a nucleation and growth process, where the Au dissolution rate was small enough to supersaturate the $\mathrm{Au}$ adatom gas into the metastable region of the phase diagram. Diffusion of Au vacancies was fast enough to allow for nucleation, before the unstable region of the phase diagram could be reached.

Apart from scientific interest, spinodal decomposition processes at surfaces are of importance also for potential applications. First attempts were undertaken to use spinodal processes in thin films for the self-organization of nanocrystals [18] or $\mathrm{Si} / \mathrm{Ge}$ islands [19]. However, in the latter systems typical length scales of the surface morphology were rather in the $100 \mathrm{~nm}$ range. In the system presented here, labyrinthine structures only a few atom diameters wide were obtained by driving a 2D Au adatom gas far from equilibrium by electrochemical means. Because of the simplicity of the system, the linearized theory by Cahn and Hilliard can be directly applied and provides a reasonable rationalization of the structures observed.

The authors thank K. G. Weil and F. Endres for discussions and comments. Technical support by G. Heyne and his co-workers is gratefully acknowledged.

*To whom correspondence should be addressed.

Electronic address: schuster@fhi-berlin.mpg.de

${ }^{\dagger}$ Present address: Department of Chemistry, Nanjing University, Nanjing 210093, People's Republic of China.

[1] P. G. Debenedetti, Metastable Liquids: Concepts and Principles (Princeton University Press, Princeton, 1996).

[2] H. Brune, Surf. Sci. Rep. 31, 121 (1998).

[3] J. D. Gunton, M.S. Miguel, and P.S. Sahni, in Phase Transitions and Critical Phenomena, edited by C. Domb and J. L. Lebowitz (Academic Press, London, 1983), Vol. 8, p. 267; K. Binder, Rep. Prog. Phys. 50, 783 (1987); A. J. Bray, Adv. Phys. 43, 357 (1994).

[4] J.W. Cahn, J. Chem. Phys. 42, 93 (1965).

[5] J.W. Cahn and J. E. Hilliard, J. Chem. Phys. 28, 258 (1958).

[6] R. Schuster, V. Kirchner, P. Allongue, and G. Ertl, Science 289, 98 (2000).

[7] R. Schuster et al., Phys. Rev. Lett. 80, 5599 (1998).

[8] H.-J. Ernst, F. Fabre, and J. Lapujoulade, Phys. Rev. Lett. 69, 458 (1992).

[9] J.-M. Wen et al., Phys. Rev. Lett. 76, 652 (1996); W.W. Pai et al., Phys. Rev. Lett. 86, 3088 (2001).

[10] P. Stoltze, J. Phys. Condens. Matter 6, 9495 (1994).

[11] T. L. Hill, An Introduction to Statistical Thermodynamics (Addison-Wesley, Reading, MA, 1962).

[12] M. Giesen and D. M. Kolb, Surf. Sci. 468, 149 (2000).

[13] A. B. Bortz, M. H. Kalos, J. L. Lebowitz, and M. A. Zendejas, Phys. Rev. B 10, 535 (1974).

[14] J. S. Langer, M. Bar-on, and H. D. Miller, Phys. Rev. A 11, 1417 (1975).

[15] F. Endres and C. Schrodt, Phys. Chem. Chem. Phys. 2, 5517 (2000).

[16] M. K. Miller et al., Acta Metall. Mater. 43, 3385 (1995).

[17] Z. Shi and J. Lipkowski, J. Electroanal. Chem. 403, 225 (1996).

[18] G. Ge and L. Brus, J. Phys. Chem. B 104, 9573 (2000); N. N. Ledentsov et al., Jpn. J. Appl. Phys. 41, 949 (2002).

[19] P. Sutter and M. G. Lagally, Phys. Rev. Lett. 84, 4637 (2000); R. M. Tromp, F. M. Ross, and M. C. Reuter, Phys. Rev. Lett. 84, 4641 (2000). 